\title{
Three identical bosons: Properties in noninteger dimensions and in external fields
}

\author{
E. Garrido $\oplus^{1, *}$ and A. S. Jensen ${ }^{2}$ \\ ${ }^{1}$ Instituto de Estructura de la Materia, IEM-CSIC, Serrano 123, E-28006 Madrid, Spain \\ ${ }^{2}$ Department of Physics and Astronomy, Aarhus University, DK-8000 Aarhus C, Denmark
}

(Received 12 June 2020; revised 23 July 2020; accepted 26 July 2020; published 18 August 2020)

\begin{abstract}
Three-body systems that are continuously squeezed from a three-dimensional space into a two-dimensional space are investigated. Such squeezing can be obtained by means of an external confining potential acting along a single axis. However, this procedure can be numerically demanding, or even undoable, especially for large squeezed scenarios. An alternative is provided by use of the dimension $d$ as a parameter that changes continuously within the range $2 \leqslant d \leqslant 3$. The simplicity of the $d$ calculations is exploited to investigate the evolution of three-body states after progressive confinement. The case of three identical spinless bosons with relative $s$ waves in three dimensions and a harmonic oscillator squeezing potential is considered. We compare results from the two methods and provide a translation between them, relating dimension, squeezing length, and wave functions from both methods. All calculations are then possible entirely within the simpler $d$ method, but simultaneously providing the equivalent geometry with the external potential.
\end{abstract}

DOI: 10.1103/PhysRevResearch.2.033261

\section{INTRODUCTION}

Specific cold atomic or molecular gases can be controlled by external fields to previously unprecedented accuracy [1,2]. This manipulation consists of two relevant ingredients, i.e., (i) the two-body effective interactions can be varied continuously between strong attraction and strong repulsion, while the system is still confined by an external trap, and (ii) the geometric space allowed by the particles can be designed at will and restricted to large volumes in three dimensions, flat or curved surfaces in two dimensions, linearity in one dimension, or anything between these geometries. The properties of the systems differ substantially depending on the confinement, which in practice can be varied by use of an external deformed potential, where one or more dimensions can be squeezed down to vanishing size.

Together with the dimension, $d$, the properties depend also on the number of particles [3]. However, so far only the $d$ dependence of the relative motion of the simplest systems has been studied by various methods [4-6]. Two particles squeezed between integer dimensions are obviously the simplest case, but beside its inherent interest, it is also necessary in investigations of three particles. One advantage is that the two masses only enter in the relative motion as the reduced mass, and only as a factor in the overall scale parameter. This is reported in previous papers using both momentum-space coordinates $[6,7]$ and ordinary space coordinates [8-10].

\footnotetext{
*e.garrido@iem.cfmac.csic.es

Published by the American Physical Society under the terms of the Creative Commons Attribution 4.0 International license. Further distribution of this work must maintain attribution to the author(s) and the published article's title, journal citation, and DOI.
}

Recently, a $d$-dependent formulation has been presented and applied to two-body systems $[9,10]$. The basic assumption is to use $d$ as a parameter that can take noninteger values, in such a way that the external squeezing potential does not appear at all but, instead, is substituted by the correspondingly modified Schrödinger equation depending on $d$ and the particle number, $N[11,12]$. The required numerical effort is similar to a standard calculation for an integer dimension, but where the external potential has disappeared. In these works, $[9,10]$, the equivalence between the $d$-dependent method and the more direct procedure working in three dimensions, including explicitly the external squeezing potential, was investigated. In the case of a squeezing harmonic oscillator potential a connection between the oscillator length and the equivalent dimension $d$ was found.

In this work we extend the method presented in Refs. $[9,10]$ to three-body systems. First, we describe in Sec. II how the hyperspherical adiabatic expansion method can be implemented to study three-body systems in a $d$ dimensional space. In Sec. III we then describe how the adiabatic expansion can be used as well to treat the same problem in a direct way, i.e., describing the system in three dimensions, but introducing explicitly the external squeezing potential. This procedure, although formally not very complicated, often leads to calculations that, especially for large squeezing, are out of numerical reach. The connection between the dimension and the external field, and the interpretation of the $d$ dimensional wave function are described in Secs. IV and V, respectively.

The two methods are applied to systems made of three identical spinless bosons and relative $s$ waves in three dimensions. In Sec. VI we specify the applied two-body potentials giving rise to different three-body scenarios. In Secs. VII and VIII the evolution of the three-body bound states after progressive squeezing is investigated for each of the two 
methods. A translation between the parameter, $d$, and the squeezing potential is investigated in Sec. IX, where the wave function in $d$ dimensions is interpreted as a deformed wave function in the ordinary three-dimensional (3D) space. We close in Sec. X with a summary and the conclusions.

\section{THREE BOSONS IN $d$ DIMENSIONS}

From a general perspective, the description of a given $N$ body system requires solving the Schrödinger equation

$$
\left[-\sum_{i=1}^{N} \frac{\hbar^{2}}{2 m_{i}} \Delta_{\boldsymbol{r}_{i}}+\frac{\hbar^{2}}{2 M} \Delta_{\boldsymbol{R}_{\mathrm{cm}}}+\sum_{i<j} V_{k}\left(\boldsymbol{r}_{i j}\right)-E_{\mathrm{Nb}}\right] \Psi=0,
$$

where $m_{i}$ and $\boldsymbol{r}_{i}$ are the mass and position vector of particle $i$, respectively, $M=\sum m_{i}$ is the total mass, and $\boldsymbol{R}_{\mathrm{cm}}$ is the $N$-body center-of-mass coordinate. The kinetic energy due to the center-of-mass motion is then explicitly removed. The potential $V_{k}\left(\boldsymbol{r}_{i j}\right)$ is the interaction between particle $i$ and particle $j$, which is assumed to depend on the relative vector, $\boldsymbol{r}_{i j}$, between the two particles. Finally, $E_{\mathrm{Nb}}$ is the total $N$-body energy.

The one-body kinetic energy operator for $N$ particles can be expressed in terms of the hyperradius, $\rho$, and all the remaining necessary angles, the hyperangles, related to the relative degrees of freedom [12]. The square, $\rho^{2}$, of the hyperradius is defined in terms of the particle coordinates and the arbitrary normalization mass, $m$, as

$$
\rho^{2}=\frac{1}{m} \sum_{i=1}^{N} m_{i}\left(\boldsymbol{r}_{i}-\boldsymbol{R}_{\mathrm{cm}}\right)^{2}=\sum_{i<j} \frac{m_{i} m_{j}}{m M}\left(\boldsymbol{r}_{i}-\boldsymbol{r}_{j}\right)^{2},
$$

which can be separated into the Cartesian coordinate contributions, i.e.,

$$
\rho^{2}=\rho_{x}^{2}+\rho_{y}^{2}+\rho_{z}^{2} .
$$

The hyperradial part of the reduced equation of motion has the usual second derivative operator and a centrifugal term, $(f-1)(f-3) /\left(4 \rho^{2}\right)$, where $f$ is the number of relative degrees of freedom. For a given $N$-body system in a general $d$-dimensional space it is then clear that $f=d(N-1)$, which results in the equation of motion in $d$ dimensions given in [12], i.e.,

$$
\begin{gathered}
{\left[-\frac{\partial^{2}}{\partial \rho^{2}}+\frac{\ell_{d, N}\left(\ell_{d, N}+1\right)+\hat{\Lambda}_{d, N}^{2}\left(\Omega_{d, N}\right)}{\rho^{2}}\right.} \\
\left.+\frac{2 m}{\hbar^{2}} \sum_{i<j} V_{k}\left(\boldsymbol{r}_{i j}\right)-\frac{2 m E_{d, N}}{\hbar^{2}}\right] \psi_{d, N}=0,
\end{gathered}
$$

where the generalized angular momentum quantum number, $\ell_{d, N}$, is given by

$$
\ell_{d, N}=\frac{f-3}{2}=\frac{1}{2}(d(N-1)-3)
$$

and where $\hat{\Lambda}_{d, N}^{2}$, which depends on the hyperangles $\Omega_{d, N}$, is the generalization to $N$ particles and $d$ dimensions of the usual hypermomentum operator [11]. The energy $E_{\mathrm{Nb}}$ in Eq. (1) is now denoted $E_{d, N}$, making explicit the dependence on the dimension. Finally, the phase-space reduced wave function, $\psi_{d, N}$, is expressed in terms of the total wave function, $\Psi_{d, N}$, as

$$
\psi_{d, N}=\rho^{\ell_{d, N}+1} \Psi_{d, N} .
$$

Once the two-body interaction potentials, $V_{k}$, are defined, different procedures can be used to reduce Eq. (4) to a set of equations depending only on the hyperradius [13]. The key in all of them consists in expanding the wave function in a certain basis set that contains the whole dependence on the hyperangles (for instance, the eigenfunctions of the $\hat{\Lambda}_{d, N}^{2}$ operator), in such a way that projection of Eq. (4) on the different basis terms immediately leads to a coupled set of differential equations for the radial function coefficients.

\section{A. The three-body case}

Being more specific, and focusing on three-body systems, $N=3$, the total three-body wave function in $d$ dimensions is obtained in this work by solving the Faddeev equations leading to the Schrödinger equation, (4). In particular, the wave function is written as

$$
\Psi_{d}=\frac{\psi_{d}}{\rho^{\frac{2 d-1}{2}}}=\frac{1}{\rho^{\frac{2 d-1}{2}}} \sum_{n} f_{n}^{(d)}(\rho) \sum_{i=1}^{3} \Phi_{n}^{(d, i)}\left(\Omega_{d}\right),
$$

where $\Psi_{d} \equiv \Psi_{d, 3}$ and where the angular functions, $\Phi_{n}^{(d, i)}$, which form a complete basis set, are the eigenfunctions, with eigenvalue $\lambda_{n}^{(d)}(\rho)$, of the $d$-dependent angular part of the Faddeev equations (see [11] for details).

Once the angular part has been solved, projection of Eq. (4) on these angular functions leads to the following coupled set of differential equations from which the radial wave functions $f_{n}^{(d)}$ in expansion (7) can be obtained:

$$
\begin{aligned}
& {\left[-\frac{\partial^{2}}{\partial \rho^{2}}+\frac{1}{\rho^{2}}\left(\lambda_{n}^{(d)}(\rho)+\frac{(2 d-3)(2 d-1)}{4}\right)-\frac{2 m E_{d}}{\hbar^{2}}\right] f_{n}^{(d)}(\rho)} \\
& =\sum_{n^{\prime}}\left(2 P_{n n^{\prime}}(\rho) \frac{\partial}{\partial \rho}+Q_{n n^{\prime}}(\rho)\right) f_{n^{\prime}}^{(d)}(\rho),
\end{aligned}
$$

where the angular eigenvalues $\lambda_{n}^{(d)}(\rho)$ enter as effective potentials, the explicit form of the coupling terms $P_{n n^{\prime}}$ and $Q_{n n^{\prime}}$ can be found in Ref. [11], and $E_{d}$ is the energy of the three-body system moving in the $d$-dimensional space $\left(E_{d} \equiv E_{d, 3}\right)$.

The method used is just the hyperspherical adiabatic expansion method, derived in detail in Ref. [11] for any arbitrary dimension $d$. The generalization of the spherical harmonics to $d$ dimensions can be found, for instance, in Appendix B of Ref. [11]. They depend on the $d-1$ angles needed to specify the direction of a given vector coordinate in $d$ dimensions. This of course makes sense for integer values of $d$, which leads to an integer number of well-defined angles (for instance, two angles for $d=3$ or one angle for $d=2$ ). However, when $d$ is allowed to take noninteger values, the definition of the angles and therefore the definition of the spherical harmonics is not obvious.

To overcome this problem we restrict ourselves to $s$ waves, i.e., zero relative orbital angular momenta between the particles. In this way the angular dependence of the spherical harmonics disappears (see the Appendix). 


\section{HARMONIC CONFINEMENT}

The method presented in the previous section appears as an alternative to the natural way of confining an $N$-particle system, which is to put it under the effect of an external potential that forces the particles to move in a limited region of space. Therefore, from the theoretical point of view, the problem to be solved is just the one given in Eq. (1) applied in three dimensions, but where both the interaction between particles, $\sum_{i<j} V_{k}$, and the trap potential, $V_{\text {trap}}$, have to be included.

An important point to keep in mind is that the energy $E_{\mathrm{Nb}}$ in Eq. (1) is now the total $N$-body energy, in such a way that the energy of the squeezed system requires subtraction of the (diverging for $d \rightarrow 2$ ) zero-point energy of an $N$-body system trapped by the potential $V_{\text {trap }}$.

In this work we consider an external harmonic oscillator potential acting along the $z$ direction. The frequency of the potential is written as

$$
\omega=\frac{\hbar}{m_{\omega} b_{\mathrm{ho}}^{2}},
$$

where $m_{\omega}$ is some arbitrary mass, and $b_{\text {ho }}$ is referred to as the harmonic oscillator length parameter. Obviously, the smaller $b_{\text {ho }}$, the more confined the particles, and eventually, for $b_{\mathrm{ho}}=0$ the particles can move only in a two-dimensional (2D) space.

The relative trap potential (the center-of-mass part is separated and omitted) can therefore be written as

$$
V_{\text {trap }}=\frac{1}{2} \omega^{2} \sum_{i=1}^{N} m_{i}\left(z_{i}-Z_{\mathrm{cm}}\right)^{2}
$$

where $z_{i}$ and $Z_{\mathrm{cm}}$ are the $z$ components of $\boldsymbol{r}_{i}$ and $\boldsymbol{R}_{\mathrm{cm}}$, respectively, and where $Z_{\mathrm{cm}}$, together with the kinetic energy term $\hbar^{2} \Delta_{\boldsymbol{R}_{\mathrm{cm}}} / 2 M$ in Eq. (1), is introduced to remove the contribution from the center-of-mass motion.

The squeezing potential can be written in a more compact way as

$$
V_{\text {trap }}=\frac{1}{2} m \omega^{2} \rho_{z}^{2}
$$

where $m$ is the arbitrary normalization mass introduced in Eq. (2), and $\rho_{z}^{2}$ refers to the $z$ contribution of the square of the generalized hyperradius vector size defined in Eqs. (2) and (3).

For this particular case, and making more specific the discussion above, the energy of the squeezed system is given by $E_{\mathrm{ext}}=E_{\mathrm{Nb}}-E_{\mathrm{ho}}$, where $E_{\mathrm{ho}}=(N-1) \hbar \omega / 2$ is the zeropoint energy in the one-dimensional (1D) squeezed oscillator for $N-1$ relative degrees of freedom.

\section{A. The three-body case}

Let us focus now on the three-body case, and let us assume an external harmonic oscillator one-body squeezing potential which, as in Eqs. (10) and (11), acts along the $z$ axis. From the definition of the $\boldsymbol{x}$ and $\boldsymbol{y}$ Jacobi coordinates [11], it is not difficult to see that the trap potential felt by the three particles can be written as

$$
\begin{aligned}
\frac{1}{2} \omega^{2} \sum_{i=1}^{3} m_{i} r_{i}^{2} \cos ^{2} \theta_{i} & =\frac{1}{2} m \omega^{2} x^{2} \cos ^{2} \theta_{x} \\
+\frac{1}{2} m \omega^{2} y^{2} \cos ^{2} \theta_{y} & +\frac{1}{2} M \omega^{2} R_{\mathrm{cm}}^{2} \cos ^{2} \theta_{\mathrm{cm}},
\end{aligned}
$$

where $\theta_{x}$ and $\theta_{y}$ are the polar angles associated with the $\boldsymbol{x}$ and $\boldsymbol{y}$ Jacobi coordinates, respectively. Therefore, after removal of the three-body center-of-mass motion the total trap potential takes the form

$$
V_{\text {trap }}\left(x, y, \theta_{x}, \theta_{y}\right)=\frac{1}{2} m \omega^{2} x^{2} \cos ^{2} \theta_{x}+\frac{1}{2} m \omega^{2} y^{2} \cos ^{2} \theta_{y},
$$

which is nothing but the particularization to three particles of Eq. (11).

A more convenient way of writing the trap potential can be obtained by working from the beginning in the three-body center of mass. This means that all coordinates are measured relative to the center of mass, $\boldsymbol{R}_{\mathrm{cm}}$. In turn, this implies that the coordinate $\boldsymbol{r}_{i}-\boldsymbol{R}_{\mathrm{cm}}$ corresponding to particle $i$ is proportional to the $\boldsymbol{y}$ Jacobi coordinate in the Jacobi set $i$. Formally, we can then insert $\boldsymbol{R}_{\mathrm{cm}}=0$ and, in the center-of-mass system, arrive at (see [11])

$$
\boldsymbol{r}_{i}=\sqrt{\frac{m}{m_{i}}} \sqrt{\frac{m_{j}+m_{k}}{m_{i}+m_{j}+m_{k}}} \boldsymbol{y}_{i},
$$

from which the potential in Eqs. (12) or (13), can also be written as

$$
V_{\text {trap }}=\sum_{i=1}^{3} V_{\text {trap }}^{(i)}=\frac{1}{2} m \omega^{2} \sum_{i=1}^{3} \frac{m_{j}+m_{k}}{m_{i}+m_{j}+m_{k}} y_{i}^{2} \cos ^{2} \theta_{y_{i}} .
$$

This last form of the squeezing potential is particularly useful when, instead of solving directly the Schrödinger equation, (1), the equation is split into its three Faddeev components.

The numerical procedure will be the same as the one shown in the previous section, i.e., we solve the Faddeev equations in coordinate space by means of the hyperspherical adiabatic expansion method described in Ref. [11]. The full calculation is now performed in three dimensions, and therefore the threebody wave function will be written as in Eq. (7) but with $d=$ 3 , that is,

$$
\Psi_{\mathrm{ext}}=\frac{1}{\rho^{5 / 2}} \sum_{n} f_{n}(\rho) \sum_{i=1}^{3} \Phi_{n}^{(i)}\left(\rho, \Omega_{i}\right),
$$

where $\rho$ is the hyperradius and $\Omega_{i}$ collects the five hyperangles associated with the Jacobi coordinates $\left\{\boldsymbol{x}_{i}, \boldsymbol{y}_{i}\right\}$, where $i$ runs over the three possible Jacobi sets [11].

Again, the angular functions $\Phi_{n}^{(i)}$ are obtained as the eigenfunctions of the angular part of the Faddeev equations, and as in the previous section, the radial wave functions $f_{n}(\rho)$ in the expansion, Eq. (16), are obtained after solving the coupled set of radial equations (8), but now particularized for $d=3$. The energy obtained in this way, $E_{3 \mathrm{~b}}$, is the total energy, system plus external field, in such a way that the energy of the confined system will be obtained after subtraction of the harmonic oscillator energy, i.e., $E_{\text {ext }}=E_{3 \mathrm{~b}}-E_{\mathrm{ho}}$, which, in our case of squeezing two coordinates along one direction [see Eq. (13)], means $E_{\mathrm{ext}}=E_{3 \mathrm{~b}}-\hbar \omega$. 
As shown in Ref. [11], the angular functions $\Phi_{n}^{(i)}$ are obtained after expanding them in terms of the hyperspherical harmonics. Calculation of the $\rho$-dependent coefficients in this expansion requires calculation of the matrix elements of the full potential in between all the hyperspherical harmonics included in the basis set. Due to the presence of the squeezing term, (15), the calculation of these matrix elements involves calculation of the integral

$$
\begin{aligned}
& W_{\ell_{x} \ell_{y} L M}^{\ell_{x}^{\prime} \ell_{y}^{\prime} L^{\prime} M^{\prime}}=\int d \Omega_{x} d \Omega_{y}\left[Y_{\ell_{x}}^{*}\left(\Omega_{x}\right) \otimes Y_{\ell_{y}}^{*}\left(\Omega_{y}\right)\right]^{L M} \\
& \times \cos ^{2} \theta_{y}\left[Y_{\ell_{x}^{\prime}}\left(\Omega_{x}\right) \otimes Y_{\ell_{y}^{\prime}}\left(\Omega_{y}\right)\right]^{L^{\prime} M^{\prime}},
\end{aligned}
$$

where $\ell_{x}$ and $\ell_{y}$ are the orbital angular momenta associated with the Jacobi coordinates $\boldsymbol{x}$ and $\boldsymbol{y}$, respectively, which couple to the total angular momentum $L$. For simplicity in the notation, we have assumed spinless particles, although the generalization to particles with nonzero spin is straightforward.

The integral in Eq. (17) is analytical, and it takes the form

$$
\begin{aligned}
W_{\ell_{x} \ell_{y} L M}^{\ell_{x}^{\prime} \ell_{y}^{\prime} L^{\prime} M^{\prime}} & \\
= & \delta_{\ell_{x} \ell_{x}^{\prime}} \delta_{M M^{\prime}} \sum_{\tilde{L}}(-1)^{\ell_{x}+M}(2 \tilde{L}+1) \hat{L} \hat{L}^{\prime} \hat{\ell}_{y} \hat{\ell}_{y}^{\prime}\left(\begin{array}{ccc}
1 & 1 & \tilde{L} \\
0 & 0 & 0
\end{array}\right)^{2} \\
& \times\left(\begin{array}{ccc}
\ell_{y} & \tilde{L} & \ell_{y}^{\prime} \\
0 & 0 & 0
\end{array}\right)\left(\begin{array}{ccc}
L & \tilde{L} & L^{\prime} \\
-M & 0 & M
\end{array}\right)\left\{\begin{array}{ccc}
L & \tilde{L} & L^{\prime} \\
\ell_{y}^{\prime} & \ell_{x} & \ell_{y}
\end{array}\right\},(18)
\end{aligned}
$$

where $\hat{\ell}$ means $\sqrt{2 \ell+1}$.

Therefore, the integral, Eq. (17), or, in other words, the trap potential, Eq. (15), mixes the relative angular momenta $\ell_{y}$ and $\ell_{y}^{\prime}$ and the total angular momenta $L$ and $L^{\prime}$, which is then not a good quantum number (unless the trap potential is equal to 0 ). The projections $M$ and $M^{\prime}$ are not mixed, and their conserved value determines the $2 \mathrm{D}$ angular momentum after an infinite squeezing of the particles along the $z$ axis.

In this work we consider the case of three identical spinless bosons, and for the case of no squeezing only $s$ waves are considered. In other words, in three dimensions the system will have quantum numbers $L=0$ and $M=0$, which implies that, throughout the squeezing process, the conserved quantum number $M$ will be equal to 0 .

\section{TRAP VERSUS THE $d$ PARAMETER}

The practical use of the $d$ formalism described in Sec. II depends on how to relate to parameters used in the laboratory setup. A universal connection was established for any two-body system squeezed from three to two dimensions by an external one-body oscillator field $[9,10]$. The provided relationship then allows us to use the parameter $d$ in the calculation and uniquely relate to an oscillator frequency or length parameter, or, in principle, vice versa.

We would like to generalize to three-body systems, but the degrees of freedom and related structures are now much larger. To begin this search we start with the very general formulation of $N$ two-body interacting particles. As described in the previous two sections, the controlling equations are described in terms of hyperspherical coordinates, where the hyperradius is the most important coordinate in a widely applicable expression. The reason is that, if the hyperangles were important, the internal $N$-body structure would influence the connection. This in turn can only appear through a complicated interpretation of the "spherical" wave function in the $d$ calculation.

To understand the relation between $d$ and $b_{\text {ho }}$ a little better, we use a simple model with an oscillator as the internal two-body interaction. Therefore, the sum of the two-body interactions entering in Eqs. (1) and (4) takes the form

$$
\begin{aligned}
\sum_{i<j} V_{k}\left(\boldsymbol{r}_{i j}\right) & =\frac{\omega_{\mathrm{pp}}^{2}}{2 M} \sum_{i<j} m_{i} m_{j}\left(\boldsymbol{r}_{i}-\boldsymbol{r}_{j}\right)^{2} \\
& =\frac{1}{2} m \omega_{\mathrm{pp}}^{2} \rho^{2},
\end{aligned}
$$

where Eq. (2) has been used and where $\omega_{\mathrm{pp}}$ describes the strength of the given particle-particle interaction.

Equations (1) and (4) are then pure harmonic oscillator equations, and the corresponding energy solutions for the $d$ calculation and the calculation with an external field, respectively, are [3]

$$
E_{d}=\hbar \omega_{\mathrm{pp}}\left(\ell_{d, N}+3 / 2\right)=\frac{1}{2} d(N-1) \hbar \omega_{\mathrm{pp}},
$$

where Eq. (5) has been used, and

$$
\begin{aligned}
E_{\mathrm{ext}}= & \hbar \omega_{\mathrm{pp}}(N-1) \\
& +\frac{(N-1)}{2} \hbar \sqrt{\omega_{\mathrm{pp}}^{2}+\omega^{2}}-\hbar \omega \frac{(N-1)}{2},
\end{aligned}
$$

where the first term is from the two nonsqueezed perpendicular directions, the second term is from the squeezed direction, and the last term removes the diverging zero-point energy. The factor $N-1$ in all terms refers to the number of relative degrees of freedom for $N$ particles. The two limits $\omega=0$ and $\omega=\infty$ produce the oscillator results corresponding to $d=3$ and $d=2$.

If the two procedures are assumed to be equivalent, we then must have that the expressions in Eqs. (20) and (21) have to be equal, which, after division throughout by $\hbar \omega_{\mathrm{pp}}$, results in

$$
d=2+\sqrt{1+\omega^{2} / \omega_{\mathrm{pp}}^{2}}-\omega / \omega_{\mathrm{pp}}
$$

and

$$
\frac{\omega}{\omega_{\mathrm{pp}}}=\frac{b_{\mathrm{pp}}^{2}}{b_{\mathrm{ho}}^{2}}=-\frac{(d-1)(d-3)}{2(d-2)},
$$

where $b_{\mathrm{pp}}^{2}=\hbar /\left(m \omega_{\mathrm{pp}}\right)$ and $m_{\omega}$ in Eq. (9) is taken as $m_{\omega}=$ $m$. This relation is independent of the number of particles, $N$, and it is only an average estimate where all structure is absent. This observation is perhaps enhanced by having three identical bosons in the ground state.

The relation given in Eq. (23) reveals the correct limit at the initial and final dimensions, which means that the squeezing length $b_{\text {ho }}$ approaches $\infty$ or 0 , respectively, for the cases of no squeezing $(d=3)$ and infinite squeezing $(d=2)$. Also, these results and conclusions could be achieved from working directly with the special cases of the present interest, $N=2$ and 3 .

When using the harmonic oscillator two-body potentials in Eq. (19), beside the energies, Eqs. (20) and (21), the wave 
functions, solutions of Eqs. (1) and (4), are also available for the two methods. They are very different in structure, since one is spherical but in $d$ dimensions, and the other is deformed in three dimensions. The ground states are Gaussians in both cases corresponding to oscillators.

In particular, for the $d$ calculation, the $N$-body ground-state oscillator wave function, $\Psi_{N, d}$, is a Gaussian, which is

$$
\Psi_{d, N} \propto \exp \left(-\frac{\rho^{2}}{2 b_{\mathrm{pp}}^{2}}\right),
$$

where $b_{\mathrm{pp}}$, as defined below Eq. (23), is the oscillator length associated with the two-body interaction, (19).

Similarly, for the case of confinement with an external field, the ground-state oscillator wave function, $\Psi_{\text {ext }}$, is also a Gaussian,

$$
\Psi_{\text {ext }} \propto \exp \left(-\frac{\rho_{\perp}^{2}}{2 b_{\perp}^{2}}\right) \exp \left(-\frac{\rho_{z}^{2}}{2 b_{z}^{2}}\right),
$$

where the oscillator lengths are

$$
b_{z}^{2}=\frac{\hbar}{m \sqrt{\omega_{\mathrm{pp}}^{2}+\omega^{2}}},
$$

coming from the combination of the two-body and the squeezing oscillators acting along the $z$ axis, and

$$
b_{\perp}^{2}=b_{\mathrm{pp}}^{2}=\frac{\hbar}{m \omega_{\mathrm{pp}}},
$$

which results from the two-body oscillator acting on the plane perpendicular to the $z$ axis, and where $\rho_{\perp}$ and $\rho_{z}$ are defined as

$$
\rho_{\perp}^{2}=x_{\perp}^{2}+y_{\perp}^{2}, \quad \rho_{z}^{2}=x_{z}^{2}+y_{z}^{2},
$$

with $\left\{x_{z}, y_{z}\right\}$ and $\left\{x_{\perp}, y_{\perp}\right\}$ being, respectively, the $z$ and perpendicular components of the Jacobi coordinates $\{\boldsymbol{x}, \boldsymbol{y}\}$.

Assuming equality between the wave functions in Eqs. (24) and (25) from the two methods we infer that the perpendicular length scale should remain unchanged, while the $z$ direction should change from $b_{\mathrm{pp}}$ to $b_{z}$. This results in a deformation along the $z$ axis of the $d$ wave function that reproduces the one of the external field. The deformation obviously depends on $d$ or confinement as expressed through Eqs. (22), (26), and (27).

These ground-state wave functions are exact for oscillators, and the large distance is asymptotically correct as soon as an oscillator confining trap is used. On the other hand, with the $d$ method the asymptotic wave function for a bound state falls off exponentially outside the determining short-range potential.

A short-range interaction more appropriate than the oscillator could clearly be found, e.g., a Gaussian of finite range. Instead in the next section we elaborate and improve on the above approximations.

\section{THE $\boldsymbol{d}$-DIMENSIONAL WAVE FUNCTION}

Let us now compare the two three-body wave functions, $\Psi_{d}$ in Eq. (7) and $\Psi_{\text {ext }}$ in Eq. (16), each obtained with their corresponding methods. The values of the dimension, $d$, and the external field parameter, $b_{\text {ho }}$, will be such that the groundstate energy is the same in both calculations.

The comparison between the two wave functions is done following closely the procedure used in Ref. [10] for two-body systems. The main idea, as suggested in the previous section when making Eqs. (24) and (25) equal, is to interpret the $d$ dimensional wave function, $\Psi_{d}$, as a deformed wave function in the ordinary 3D coordinate space. The Jacobi coordinates, $\boldsymbol{x}$ and $\boldsymbol{y}$, in $d$ dimensions are then redefined in the 3D space as $\tilde{\boldsymbol{x}}$ and $\tilde{\boldsymbol{y}}$, with ordinary 3D Cartesian components. However, since the squeezing is assumed to take place along the $z$ axis the deformation will take place along that axis, and the $z$ components of the $3 \mathrm{D}$ vectors $\tilde{\boldsymbol{x}}$ and $\tilde{\boldsymbol{y}}$ will be deformed. Both Jacobi coordinates will be deformed in the same way, which amounts to assuming that all the particles feel the squeezing equally.

More precisely, the modulus of the Jacobi coordinates is redefined as

$$
\begin{aligned}
& x \rightarrow \tilde{x}=\sqrt{x_{\perp}^{2}+\left(x_{z} / s\right)^{2}}, \\
& y \rightarrow \tilde{y}=\sqrt{y_{\perp}^{2}+\left(y_{z} / s\right)^{2}},
\end{aligned}
$$

from which it is possible to construct the usual hyperspherical coordinates in three dimensions, i.e., $\tilde{\rho}=\left(\tilde{x}^{2}+\tilde{y}^{2}\right)^{1 / 2}, \tilde{\alpha}=$ $\arctan (\tilde{x} / \tilde{y})$, and $\tilde{\Omega}_{\tilde{x}}$ and $\tilde{\Omega}_{\tilde{y}}$, which are the polar and azimuthal angles giving the directions of $\tilde{\boldsymbol{x}}$ and $\tilde{\boldsymbol{y}}$.

The scale parameter, $s$, controls the deformation of the wave function, in such a way that for $s=1$ the wave function is not deformed, and for $s=0$ only $x_{z}=0$ and $y_{z}=0$ are possible, and the system is therefore fully squeezed into a $2 \mathrm{D}$ space. The introduction of the parameter, $s$, and the interpretation of $\Psi_{d}$ as a deformed wave function in three dimensions makes it necessary to renormalize the wave function to $\tilde{\Psi}_{d}=$ $\Psi_{d} / C(s)$, where $C(s)$ is given by

$$
C^{2}(s)=\int d^{3} x d^{3} y\left|\Psi_{d}\left(x_{\perp}, x_{z}, y_{\perp}, y_{z}, s\right)\right|^{2} .
$$

As in Ref. [10], the wave functions, $\Psi_{\text {ext }}$ and $\tilde{\Psi}_{d}$, are compared through their overlap, i.e.,

$$
\mathcal{O}(s)=\int d^{3} x d^{3} y \Psi_{\mathrm{ext}}^{*}(\boldsymbol{x}, \boldsymbol{y}) \tilde{\Psi}_{d}\left(x_{\perp}, x_{z}, y_{\perp}, y_{z}, s\right),
$$

which can be used as a measure of the accuracy of the scaling interpretation of the initially spherical $d$-dimensional wave function. The scale parameter could temptingly be extracted as the value of $s$ maximizing $\mathcal{O}(s)$.

The deformation choice described by Eqs. (29) and (30) is just an estimate of the actual wave-function deformation, where the scale factor is taken to be constant. Other different choices are certainly possible, for instance, considering the scale factor, $s$, to be a function of $x_{z}$ and $y_{z}$. In any case the overlap described in Eq. (32) must be smaller than 1. As a general rule, the smaller the squeezing, the closer to 1 the overlap, or, in other words, the better the deformation interpretation as expressed in Eqs. (29) and (30).

It is worth emphasizing that the overlap must be unity in both limits of three dimensions (no squeezing) and two dimensions (infinite squeezing). In three dimensions it is obvious, since both wave functions are solutions of the same equation, 
Eq. (4), for $N=3$ and $d=3$. The maximum overlap between the two solutions will then obviously be equal to 1 , and no deformation of the $d$-wave function will be necessary (i.e., $s=1$ ). In two dimensions the wave functions from the two methods must also be identical, since they are solutions to the same problem in two dimensions no matter how they are obtained. The maximum overlap is then again equal to 1 but now corresponding to $s=0$. As will be shown, the problem in this case is the difficulty in reaching this limit with the external squeezing potential.

The similarity between the two wave functions, $\Psi_{\text {ext }}$ and $\tilde{\Psi}_{d}$, can be visualized by expanding $\tilde{\Psi}_{d}$ in terms of the angular eigenfunctions, $\Phi_{n}^{(i)}(\rho, \Omega)$, used to expand $\Psi_{\text {ext }}$ in Eq. (16), i.e.,

$$
\tilde{\Psi}_{d}\left(x_{\perp}, x_{z}, y_{\perp}, y_{z}, s\right)=\frac{1}{\rho^{5 / 2}} \sum_{n} \tilde{f}_{n}^{(d)}(\rho, s) \sum_{i=1}^{3} \Phi_{n}^{(i)}\left(\rho, \Omega_{i}\right),
$$

which, due to the orthogonality of the angular functions, leads to

$$
\tilde{f}_{n}^{(d)}(\rho, s)=\rho^{5 / 2} \int d \Omega \Phi_{n}^{*}(\rho, \Omega) \tilde{\Psi}_{d}\left(x_{\perp}, x_{z}, y_{\perp}, y_{z}, s\right),
$$

where $\Phi_{n}=\sum_{i} \Phi_{n}^{(i)}$ and $d \Omega$ is the usual phase space associated with the hyperangles for three particles in three dimensions. The difference between the $\Psi_{\text {ext }}$ and $\tilde{\Psi}_{d}$ is then due exclusively to the different behavior of the radial wave functions $f_{n}(\rho)$ and $\tilde{f}_{n}^{(d)}(\rho)$.

After this interpretation of the $d$-dimensional wave function, based on Eqs. (29) and (30), we have that the wave function, Eq. (24), obtained for the harmonic oscillator twobody interaction, Eq. (19), can be understood as an ordinary 3D wave function, which has the large-distance asymptotic behavior

$$
\tilde{\Psi}_{d, N} \propto \exp \left(-\frac{\tilde{\rho}^{2}}{2 b_{\mathrm{pp}}^{2}}\right)=\exp \left(-\frac{\rho_{\perp}^{2}}{2 b_{\mathrm{pp}}^{2}}\right) \exp \left(-\frac{\rho_{z}^{2}}{2 s^{2} b_{p \mathrm{p}}^{2}}\right),
$$

where $\rho_{\perp}$ and $\rho_{z}$ are given in Eq. (28).

For this wave function to equal that of Eq. (25), obtained with an external field, and keeping in mind that $b_{\mathrm{pp}}=b_{\perp}$, it is simple to see that we must have

$$
\frac{1}{s^{2}}=\frac{b_{\mathrm{pp}}^{2}}{b_{z}^{2}}=\sqrt{1+\frac{\omega^{2}}{\omega_{\mathrm{pp}}^{2}}},
$$

where Eqs. (26) and (27) have been used.

Now making use of Eq. (23), we finally obtain a crude estimate of the relation between the scale parameter, $s$, the squeezing harmonic oscillator parameter, $b_{\mathrm{ho}}$, and the dimension, $d$ :

$$
\frac{1}{s^{2}}=\frac{b_{\mathrm{pp}}^{2}}{b_{z}^{2}}=\sqrt{1+\frac{b_{\mathrm{pp}}^{4}}{b_{\mathrm{ho}}^{4}}}=\sqrt{1+\left(\frac{(d-1)(d-3)}{2(d-2)}\right)^{2}} .
$$

This estimate is clearly very simple but at the same time independent of any details referring to the squeezing process. Thus it must necessarily be an approximation when applied to large squeezing corresponding to distances influenced by the short-range interaction.
TABLE I. Strengths, $S$, of the Gaussian $\left(A_{g}\right.$ and $\left.B_{g}\right)$ and Morse $\left(A_{m}\right.$ and $\left.B_{m}\right)$ two-body potentials used in this work. For each of them we list the binding energy of the existing two-body bound states in two dimensions, $E_{2 \mathrm{D}}^{(2 \mathrm{bd})}$, and in three dimensions, $E_{3 \mathrm{D}}^{(2 \mathrm{bd})}$, as well as the corresponding 2D and 3D scattering lengths, $a_{2 \mathrm{D}}$ and $a_{3 \mathrm{D}}$. The energies and lengths are given in units of $\hbar^{2} / m b^{2}$ and $b$, respectively, where $m$ is the mass of each particle and $b$ is the range of the interaction.

\begin{tabular}{lccccc}
\hline \hline Potential & $S$ & $E_{2 \mathrm{D}}^{(2 \mathrm{bd})}$ & $a_{2 \mathrm{D}}$ & $E_{3 \mathrm{D}}^{(2 \mathrm{bd})}$ & $a_{3 \mathrm{D}}$ \\
\hline$A_{g}$ & -2.86 & -0.538 & 1.883 & $-3.301 \times 10^{-3}$ & 18.122 \\
$B_{g}$ & -14.50 & -7.918 & 3.916 & -5.075 & -0.201 \\
& & -0.134 & & & \\
$A_{m}$ & 0.95 & -0.148 & 3.536 & $-3.751 \times 10^{-3}$ & 18.122 \\
$B_{m}$ & 3.34 & -1.327 & 19.783 & -0.665 & -0.201 \\
& \multicolumn{7}{c}{$-3.971 \times 10^{-3}$} & & \\
\hline \hline
\end{tabular}

\section{RESULTS: TWO-BODY POTENTIALS}

In this work we consider three identical spinless bosons. This is a particularly simple case, since the three terms of the confining potential, Eq. (15), are then identical. This fact has the advantage that with the proper choice of the energy and length units, the equations of motion become independent of the mass of the particles. For three different masses the dependence of the squeezing potential on the mass ratios becomes unavoidable.

In particular, we take $m_{\omega}$ in Eq. (9) and the normalization mass, $m$, used to construct the Jacobi coordinates equal to each other and equal to the mass of each of the particles. The length unit will be some length characterizing the bosonboson potential, which in our case will be the range, $b$, of the short-range interaction acting between them. The energy unit will be taken equal to $\hbar^{2} / m b^{2}$. When this is done, the dependence on $m$ of Eqs. (1) and (4), or, equivalently, of Eq. (8), disappears.

In this work we consider two different shapes for the two-body potentials, a Gaussian potential and a Morse-like potential. After choosing the range of the interaction as the length unit, these two potentials can be written as $S e^{-r^{2}}$ and $S\left(e^{-2 r}-2 e^{-r}\right)$, respectively. For each shape we have taken two different potentials, whose corresponding strengths are given in the second column in Table I. These potentials give rise to a series of bound two-body states in two and three dimensions, whose corresponding binding energies, $E_{2 \mathrm{D}}^{(2 \mathrm{bd})}$ and $E_{3 \mathrm{D}}^{(2 \mathrm{bd})}$, are listed in the third and fifth columns in the table, respectively. In the fourth and sixth columns we give for each potential the scattering length in two dimensions, $a_{2 \mathrm{D}}$, and in three dimensions, $a_{3 \mathrm{D}}$ (see Ref. [11] for definitions).

The Gaussian and Morse potentials are called potentials $A_{g}, B_{g}$ and $A_{m}, B_{m}$, respectively. The main difference between them is the number of two-body bound states in two and three dimensions. Potentials $A$ have the same number of bound states, one, in two and three dimensions, whereas potentials $B$ have one bound state in three dimensions but two in two dimensions. Note that potentials $A_{g}$ and $A_{m}$, and $B_{g}$ and $B_{m}$, have the same value of the $3 \mathrm{D}$ scattering length $a_{3 \mathrm{D}}$. Potentials $A$ have a large value of $a_{3 \mathrm{D}}$, which is reflected in the small binding of the $3 \mathrm{D}$ ground state. Potentials $B$ have a quite 


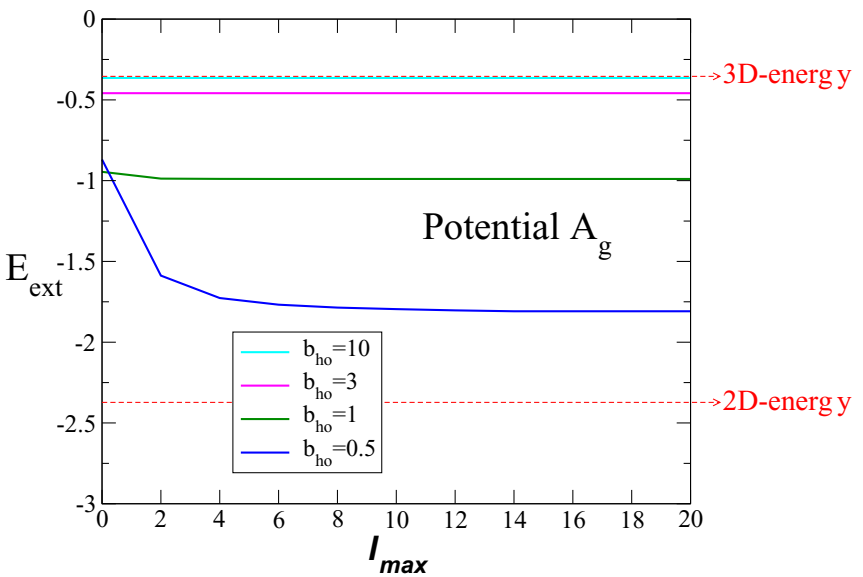

FIG. 1. For the Gaussian potential $A_{g}$ and an external squeezing harmonic oscillator field, we show the three-body ground-state binding energy, $E_{\mathrm{ext}}=E_{3 \mathrm{~b}}-E_{\mathrm{ho}}$, as a function of the maximum $\ell_{y}$ value, $\ell_{\max }$, used on each Jacobi set. The results with different values of the squeezing parameter $b_{\text {ho }}$ are shown. The lower and higher dashed horizontal lines indicate the $2 \mathrm{D}$ and $3 \mathrm{D}$ energies, respectively. The energy is in units of $\hbar^{2} / m b^{2}$, where $m$ is the mass of each particle and $b$ is the range of the interaction.

modest and negative value of $a_{3 \mathrm{D}}$, which indicates that the first excited state, although unbound, is not very far from the threshold. Potential $B_{m}$ has a positive large value of $a_{2 \mathrm{D}}$, responsible for the little binding of the first excited two-body state. Potentials $A$ are the ones called potentials II in Ref. [10], although in this work the length unit is a factor of 2 smaller than the one used in Ref. [10].

\section{RESULTS: EXTERNAL FIELD CASE}

As mentioned several times, the most direct way to investigate particle confinement consists in including the external potential in the problem to be solved. In our case this means solving the three-body problem as described in Sec. III A, where the squeezing potential enters explicitly.

However, this method presents two main problems. The first one refers to the fact that, as seen in Eq. (18), although $\ell_{x}$ (taken equal to 0 in this work) is conserved all along the squeezing process, the orbital angular momentum $\ell_{y}$ and, therefore, $L$ as well are no longer good quantum numbers. This mixing of partial waves is introduced by the squeezing potential, and in fact, the larger the squeezing of the particles the more partial waves are needed.

This is illustrated in Fig. 1, where we show for the Gaussian potential $A_{g}$ in Table I how the ground-state energy, $E_{\mathrm{ext}}=$ $E_{3 \mathrm{~b}}-E_{\mathrm{ho}}$, of the confined three-body system converges as a function of $\ell_{\max }$, where $\ell_{\max }$ refers to the maximum value of $\ell_{y}=L$ included in each of the Faddeev components. The lower and higher dashed horizontal lines in the figure indicate the ground-state energy after a pure $2 \mathrm{D}$ and a pure $3 \mathrm{D}$ calculation, respectively. The results are shown for different values of the squeezing parameter $b_{\mathrm{ho}}$.

As we can see, for sufficiently large values of $b_{\text {ho }}$ the convergence is very fast and just a few $\ell_{y}$ components, very often only one, are enough. Eventually, for very large $b_{\text {ho }}$ the

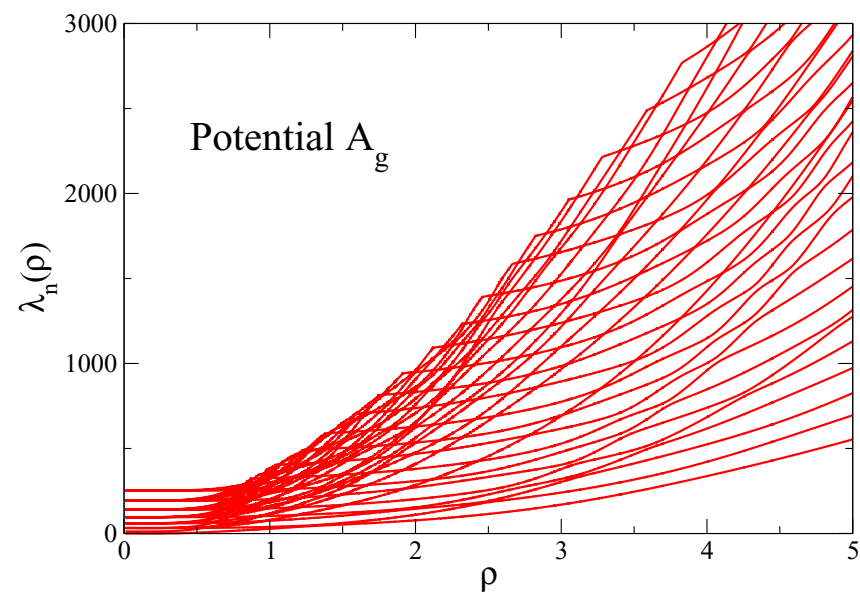

FIG. 2. Thirty lowest $\lambda_{n}$ functions for potential $A_{g}$ with a squeezing external harmonic oscillator field of oscillator length $b_{\mathrm{ho}}=0.2$, and $\ell_{\max }=14$.

3D energy is recovered. With decreasing $b_{\text {ho }}$ we observe that higher and higher values of $\ell_{\max }$ are needed. For instance, for $b_{\text {ho }}=0.5$ the components with $\ell_{y}$ up to at least 14 are necessary to get convergence. For a sufficiently small value of $b_{\text {ho }}$ the converged energy should eventually match the 2D energy indicated by the lower dashed straight line in the figure.

However, getting the same kind of curve for smaller values of $b_{\mathrm{ho}}$ is not simple. This is due to the second problem to be faced when solving the three-body equations with an external potential, which refers to the convergence of the expansion in Eq. (16). The number of terms needed for convergence increases with the confinement of the particles. This should not be a big problem in itself, except for the fact that when the squeezing of the particles increases (small $b_{\text {ho }}$ values), the number of crossings between the different $\lambda_{n}$ functions entering in Eq. (8) becomes eventually too high, which increases the computing time dramatically. This problem is actually enhanced by the fact that, as explained above, the number of required partial waves increases as well with the confinement.

As an illustration we show in Fig. 2 the 30 lowest $\lambda_{n}(\rho)$ functions for potential $A_{g}, b_{\mathrm{ho}}=0.2$, and $\ell_{\max }=14$. To deal with such a huge number of crossings is very time-consuming and makes this method rather inefficient close to two dimensions. Towards this limit the method of correlated Gaussians [4] might, for example, be used, although the advantage of very similar basis functions would then also disappear. The choice is then between using different methods in the two limits or the same method with inherent loss of efficiency in one of the limits.

The conclusion is therefore that to include explicitly the external potential as described in Sec. III A is not very convenient in the case of large squeezing. To reach convergence for low values of the squeezing parameter becomes at some point too troublesome. In Fig. 3 we show the computed converged energies of the three-body ground state for the four potentials

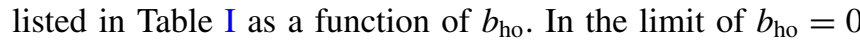
the $2 \mathrm{D}$ energies indicated by the arrows in the figure should be reached. 


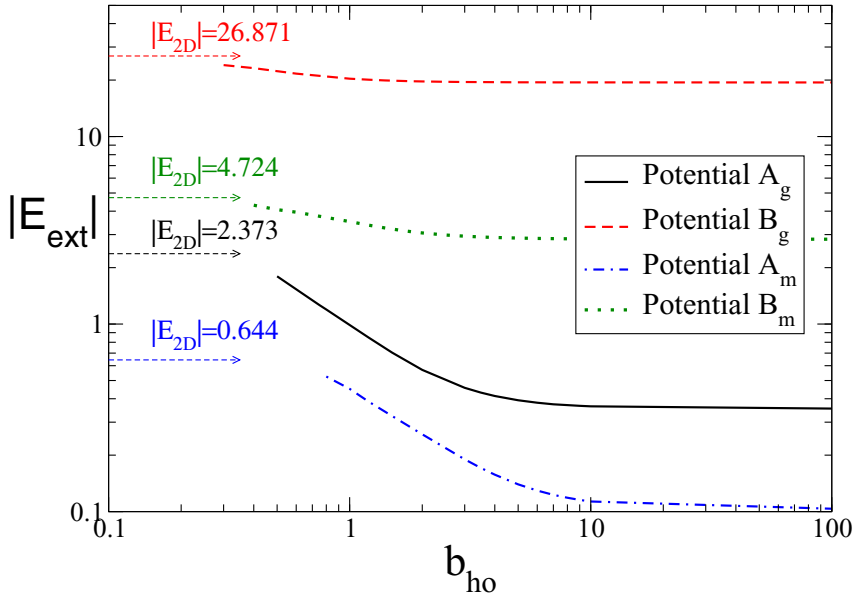

FIG. 3. For the case of squeezing with an external harmonic oscillator field, the absolute value of the converged three-body ground-state energies (in units of $\hbar^{2} / m b^{2}$ ) for the Gaussian potentials $A_{g}$ (solid line) and $B_{g}$ (dashed line) and the Morse potentials $A_{m}$ (dot-dashed line) and $B_{m}$ (dotted line), as a function of $b_{\text {ho }}$ (in units of the range of the interaction). Arrows indicate the $2 \mathrm{D}$ energy that should be reached for $b_{\mathrm{ho}}=0$.

\section{RESULTS: THE $\boldsymbol{d}$ CALCULATION}

Performing the three-body calculations as described in Sec. II, where the external potential does not enter and the dimension $d$ is treated as a parameter, is certainly much simpler than performing the calculations shown in the previous section. In Table II we give the computed energies and rootmean-square (rms) radii in two and three dimensions for all the three-body bound states obtained after solving the coupled Eqs. (8) with the two-body potentials listed in Table I. The energies, $E_{2 \mathrm{D}}$ and $E_{3 \mathrm{D}}$, and the rms values, $r_{2 \mathrm{D}}$ and $r_{3 \mathrm{D}}$, refer to the results obtained with $d=2$ and $d=3$, respectively. As we can see, whereas potentials $A_{g}, A_{m}$, and $B_{m}$ have the same number, two, of bound three-body states in three dimensions

TABLE II. For $d=2$ and $d=3$, the three-body energies and root-mean-square radii, $r=\left\langle\rho^{2} / 3\right\rangle^{1 / 2}$, for all the bound states obtained with the potentials listed in Table I. The subscripts $2 \mathrm{D}$ and $3 \mathrm{D}$ refer to the results obtained for $d=2$ and $d=3$, respectively. As done throughout the text, the energies are given in units of $\hbar^{2} / m b^{2}$, where $m$ is the mass of each of the particles (and the normalization mass used to construct the Jacobi coordinates), and $b$ is the range of the two-body interaction, which is taken as the length unit.

\begin{tabular}{lcccr}
\hline \hline Potential & $E_{2 \mathrm{D}}$ & $r_{2 \mathrm{D}}$ & $E_{3 \mathrm{D}}$ & \multicolumn{1}{c}{$r_{3 \mathrm{D}}$} \\
\hline$A_{g}$ & -2.373 & 0.591 & -0.354 & 1.081 \\
& -0.556 & 3.339 & $-6.109 \times 10^{-3}$ & 10.280 \\
$B_{g}$ & -26.871 & 0.304 & -19.426 & 0.390 \\
& -14.622 & 0.518 & -9.050 & 0.632 \\
& -7.990 & 1.678 & & \\
$A_{m}$ & -0.644 & 1.145 & -0.104 & 2.201 \\
& -0.160 & 4.695 & $-4.808 \times 10^{-3}$ & 15.744 \\
$B_{m}$ & -4.724 & 0.613 & -2.834 & 0.848 \\
& -2.202 & 1.220 & -1.087 & 1.628 \\
\hline \hline
\end{tabular}
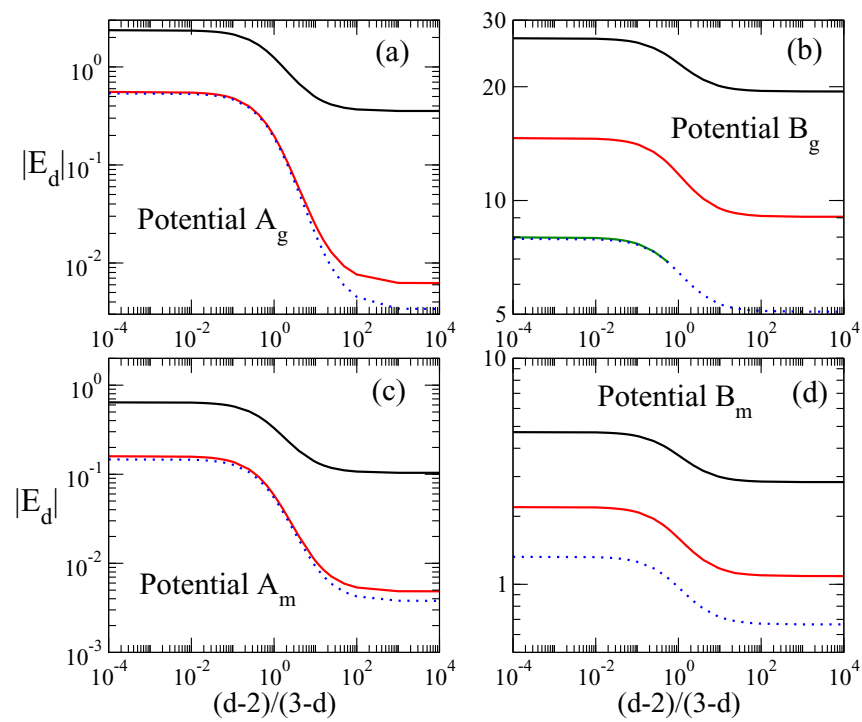

FIG. 4. With the $d$ method, the absolute value of the bound-state three-body energies obtained with potentials (a) $A_{g}$, (b) $B_{g}$, (c) $A_{m}$, and (d) $\mathrm{B}_{m}$ in Table I as a function of $(d-2) /(3-d)$, where $d$ runs within the range $2 \leqslant d \leqslant 3$. For each of the potentials, the dotted blue curve is the absolute value of the lowest bound two-body state energy.

as in two dimensions, potential $B_{g}$ has one bound state less in three dimensions, two, than in two dimensions, three.

In Fig. 4 we show the absolute value of the bound threebody energies obtained with potentials $A_{g}, B_{g}, A_{m}$, and $B_{m}$ [Figs. 4(a)-4(d), respectively] as a function of $(d-2) /(3-$ $d$ ), where $d$ is the dimension running within the range $2 \leqslant$ $d \leqslant 3$. The choice of the abscissa coordinate as $(d-2) /(3-$ $d$ ) has been made to facilitate the comparison with Fig. 3, since in both figures values of the abscissa coordinate equal to 0 and $\infty$ correspond, respectively, to maximum squeezing of the system into two dimensions and no squeezing (three dimensions). In the figure the dotted blue curve shows, for each of the potentials and also as a function of $(d-2) /(3-$ $d)$, the absolute value of the lowest two-body bound-state energy.

As a general rule, when squeezing from $d=3$ to $d=2$ the three-body system becomes progressively more and more bound. This is clearly seen for all the potentials when moving from the right part in each panel $(d=3)$ to the left part $(d=$ 2 ). This is a reflection of the lower centrifugal barrier in two dimensions than in three dimensions.

In the case of potentials $A_{g}$ and $A_{m}$ [Figs. 4(a) and 4(c)], the red curve corresponding to the excited three-body bound state approaches the dotted curve representing the bound twobody energy. In fact, very soon the three-body excited state is just slightly more bound than the two-body state, therefore representing a boson very weakly bound with respect to the two-body bound state.

For potential $B_{g}$ [Fig. 4(b)] a new bound state shows up for $d \approx 2$.4. It appears from the threshold corresponding to the bound two-body state and the third boson. From that point and up to $d=2$ the third three-body bound state follows closely the two-body binding energy (dotted curve). Thus, as 
for potentials $A$, the last excited state corresponds to a very weakly bound boson with respect to the two-body bound state.

For potential $B_{m}$ the situation seems to be different compared to the other potentials, since in this case there is no three-body bound state approaching the two-body bound-state curve (dotted curve). However, the behavior of the three-body spectrum for this potential is actually very similar to that for potential $B_{g}$ in Fig. 4(b). The only difference is that the dimension at which the third bound state appears from the two-body threshold is $d \approx 1.9$, therefore lying out of the graph limits. It would certainly be possible to extend the investigation to confinement scenarios up to dimensions smaller than 2 , eventually up to $d=1$ or even smaller, but this is left for a future work.

\section{WAVE FUNCTIONS}

In Sec. IV the relation between the harmonic oscillator parameter, $b_{\mathrm{ho}}$, and the dimension, $d$, was discussed. In particular, the relation in Eq. (23) was found for the simple case where the particle-particle interaction is assumed to be a harmonic oscillator potential with length parameter $b_{\mathrm{pp}}$. This length, $b_{\mathrm{pp}}$, is also the rms radius of the three-body system in three dimensions, whereas in two dimensions the rms radius is $r_{2 \mathrm{D}}=b_{\mathrm{pp}} \sqrt{2 / 3}$, which permits us to rewrite Eq. (23) as

$$
\frac{b_{\mathrm{ho}}}{r_{2 \mathrm{D}}}=\sqrt{\frac{3(d-2)}{(d-1)(3-d)}} .
$$

Instead of this simple expression, the full numerical solution from the Gaussian and Morse short-range interactions can be found, and the energies compared. In the same way that Eq. (23) has been obtained by making $E_{d}$ and $E_{\text {ext }}$ equal in Eqs. (20) and (21), we can use Figs. 3 and 4 to obtain numerically the relation between $d$ and $b_{\text {ho }}$, at least for the $b_{\text {ho }}$ values for which, as shown in Fig. 3, the energy $E_{\text {ext }}$ can be obtained.

The results are shown in Fig. 5 by the solid, dashed, dot-dashed, and dot-dot-dashed curves for potentials $A_{g}, B_{g}$, $A_{m}$, and $B_{m}$, respectively. They have been obtained using the energies of the three-body ground state. The numerical curves are compared with the analytical expression given by Eq. (38), which is shown by the dotted curve.

As we can see, the estimate in Eq. (38) agrees reasonably well with the calculations in those regions where the numerical calculation with the external potential has been possible. This is especially true for potentials $A_{g}, B_{g}$, and $A_{m}$, whereas for potential $B_{m}$ a somewhat bigger discrepancy is observed for large squeezing. In any case, for those small values of the squeezing parameter, $b_{\text {ho }}$, the complications inherent to the numerical calculations with the external potential can be a source of inaccuracy for $E_{\text {ext }}$. Furthermore, since $b_{\text {ho }}=0$ must necessarily correspond to $d=2$, we can consider expression (38) a reliable translation to the parameter, $d$, in relatively easy calculations from a given small value of the squeezing parameter, $b_{\text {ho }}$

As shown in Sec. V, the equivalence between the threebody wave functions with the two calculations can be directly compared through the overlap in Eq. (32), where $\Psi_{\text {ext }}$ is the wave function obtained with the external squeezing potential,

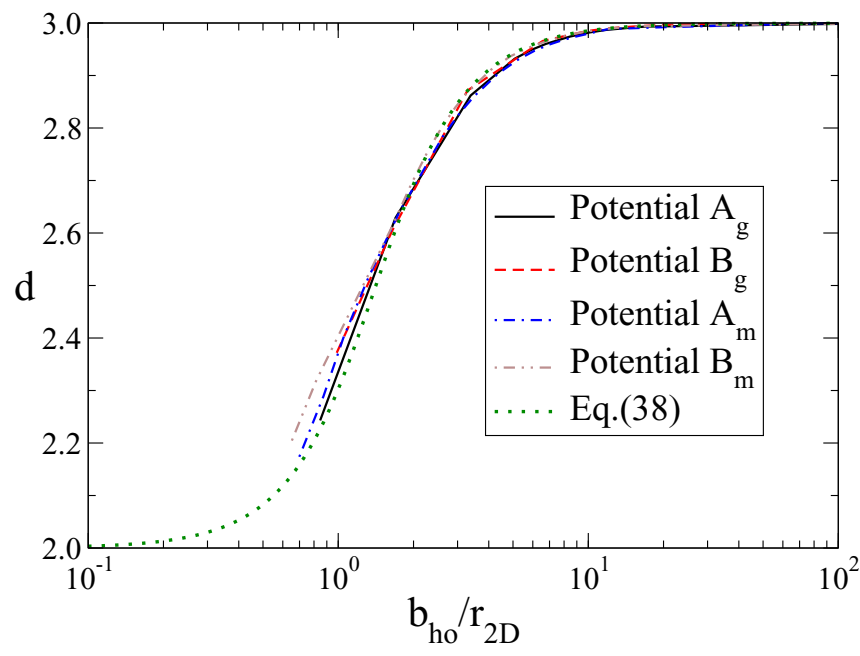

FIG. 5. Numerical relation between the dimension $d$ and the harmonic oscillator parameter $b_{\text {ho }}$ obtained after making $E_{\text {ext }}$ in Fig. 3 and the ground-state energy $E_{d}$ in Fig. 4 equal. The oscillator parameter $b_{\text {ho }}$ is normalized to the root-mean-square radius of the $2 \mathrm{D}$ three-body calculation. The cases of potentials $A_{g}, B_{g}, A_{m}$, and $B_{m}$ are shown by the solid, dashed, dot-dashed, and dot-dot-dashed curves, respectively. These results are compared with the estimate given in Eq. (38), which is shown by the dotted curve.

and $\tilde{\Psi}_{d}$ is the one obtained with the $d$ calculation after the transformation in Eqs. (29) and (30), and the subsequent renormalization. Obviously, the values of $b_{\mathrm{ho}}$ and $d$ have to be such that they both give rise to the same ground-state threebody energy. As discussed in Sec. V, this overlap permits us to extract the scale parameter, $s$, in the transformation in Eqs. (29) and (30) as the value that maximizes the overlap.

In Fig. 6 we show the overlap $\mathcal{O}(s)$ as a function of the scale parameter, $s$, for the four potentials considered in this work. Several different values of the squeezing parameter, $b_{\text {ho }}$, have been chosen, and for each of them we determine, according to Fig. 5, the value of the dimension, $d$, giving rise to the same ground-state binding energy. As shown in Fig. 6, in all cases the curves show a well-defined maximum, which determines the value of the scale parameter, $s$, to be used in the $d$ function $\tilde{\Psi}_{d}$.

Figure 6(a) shows the results for potential $A_{g}$. As we can see, for $b_{\mathrm{ho}}=1.5$, which corresponds to $d=2.783$, we obtain a scale parameter of $s=0.96$, which indicates that the deformation of the $d$-calculated wave function produced by the squeezing is in this case rather modest. For larger squeezing (smaller values of $b_{\mathrm{ho}}$ ), the scale parameter starts decreasing, reaching the values of $s=0.91, s=0.86$, and $s=0.78$ for $b_{\mathrm{ho}}=1.2(d=2.704), b_{\mathrm{ho}}=1.0(d=2.628)$, and $b_{\mathrm{ho}}=0.8$ $(d=2.523)$, respectively. These results are collected in the second column in Table III.

The deformation of the $d$-calculated wave function produced by a given squeezing is very sensitive to the size of the three-body system. For instance, as shown in Table II, for potential $B_{g}$ the $3 \mathrm{D}$ ground state is about three times smaller than the one for potential $A_{g}$. For this reason one can intuitively think that a larger squeezing (smaller $b_{\text {ho }}$ ) will be necessary in order to get a similar deformation of the 

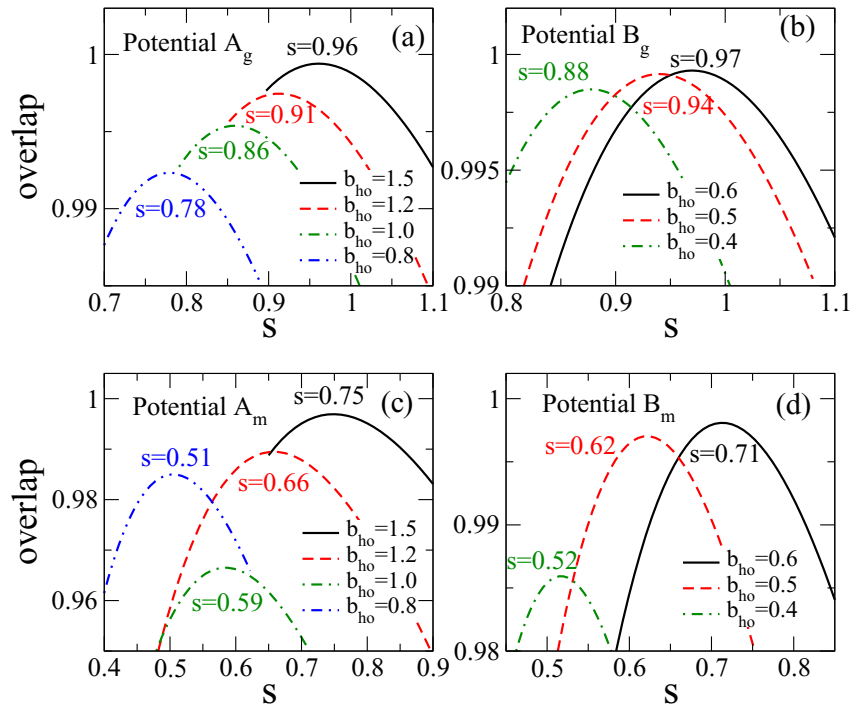

FIG. 6. Overlap $\mathcal{O}(s)$ as a function of the scale parameter $s$ for potentials (a) $A_{g}$, (b) $B_{g}$, (c) $A_{m}$, and (d) $B_{m}$ and different values of the external potential parameter $b_{\mathrm{ho}}$. For each $b_{\mathrm{ho}}$ the dimension $d$ is the one giving rise to the same ground-state binding energy. In each case we indicate the value of $s$ corresponding to the maximum overlap.

wave function. This is actually shown in Fig. 6(b), where the overlap $\mathcal{O}(s)$ for potential $B_{g}$ is shown (the corresponding $s$ and $d$ values are given in the third column in Table III). As we can see, even for a squeezing parameter $b_{\text {ho }}=0.6$ ( $d=2.694$ ) the scale parameter is still not too far from 1 , whereas for $b_{\mathrm{ho}}=0.5(d=2.604)$ and $b_{\mathrm{ho}}=0.4(d=2.489)$ the corresponding values of $s$ are similar to those obtained for potential $A_{g}$ [Fig. 6(a)] when $b_{\text {ho }}=1.2$ and $b_{\text {ho }}=1.0$, respectively.

In Figs. 6(c) and 6(d) we show the same results for the Morse-like potentials $A_{m}$ and $B_{m}$, respectively, and the values of $s$ and $d$ are also listed in the fourth and fifth columns in Table III. The general behavior is similar to that found for the Gaussian potentials. The three-body system is again about three times larger in three dimensions with potential $A_{m}$ than with potential $B_{m}$. The result is therefore that similar deformation, i.e., similar values of the scale parameter $s$, requires less squeezing, a larger value of $b_{\text {ho }}$, with potential $A_{m}$ than

TABLE III. For each of the potentials and each of the selected values of $b_{\text {ho }}$ shown in Fig. 6 , the corresponding values of the scale parameter $s$ that maximizes the overlap in Eq. (32) and the dimension $d$ giving rise to the same ground-state binding energy.

\begin{tabular}{|c|c|c|c|c|c|c|c|c|}
\hline \multirow[b]{2}{*}{$b_{\mathrm{ho}}$} & \multicolumn{2}{|c|}{ Potential $A_{g}$} & \multicolumn{2}{|c|}{ Potential $B_{g}$} & \multicolumn{2}{|c|}{ Potential $A_{m}$} & \multicolumn{2}{|c|}{ Potential $B_{m}$} \\
\hline & $s$ & $d$ & $s$ & $d$ & $s$ & $d$ & $s$ & $d$ \\
\hline 0.4 & & & 0.88 & 2.489 & & & 0.52 & 2.205 \\
\hline 0.5 & & & 0.94 & 2.604 & & & 0.62 & 2.318 \\
\hline 0.6 & & & 0.97 & 2.694 & & & 0.71 & 2.383 \\
\hline 0.8 & 0.78 & 2.523 & & & 0.51 & 2.173 & & \\
\hline 1.0 & 0.86 & 2.628 & & & 0.59 & 2.289 & & \\
\hline 1.2 & 0.91 & 2.704 & & & 0.66 & 2.400 & & \\
\hline 1.5 & 0.96 & 2.783 & & & 0.75 & 2.511 & & \\
\hline
\end{tabular}

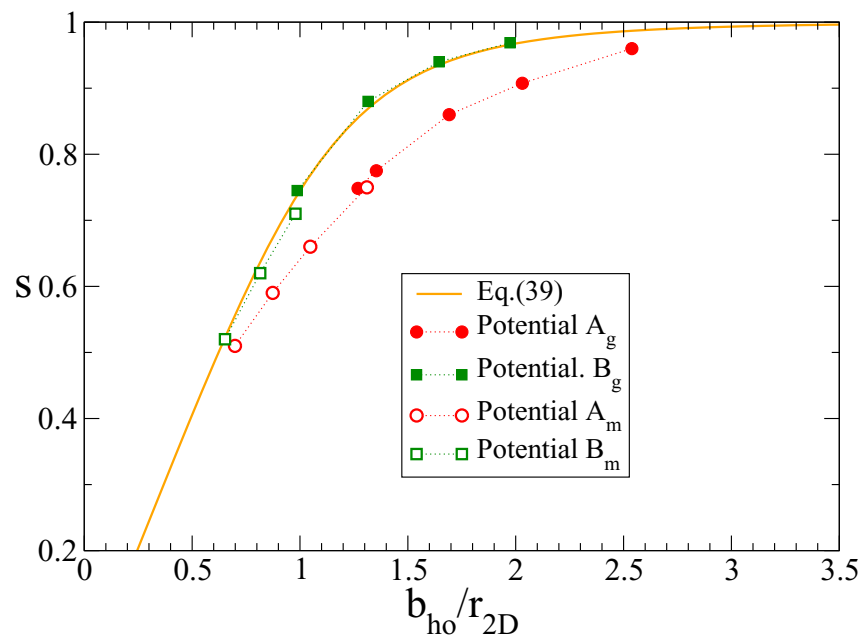

FIG. 7. Scale parameter $s$ as a function of $b_{\mathrm{ho}} / r_{2 \mathrm{D}}$ for the four potentials used in this work. The solid curve shows the estimate given in Eq. (39).

with potential $B_{m}$. The same thing occurs when comparing potentials $A_{m}$ and $A_{g}$ and potentials $B_{m}$ and $B_{g}$. Since the Morse 3D states are clearly larger than the corresponding Gaussian counterparts, we again find that for equal values of $b_{\text {ho }}$ the larger systems, those obtained with potentials $A_{m}$ and $B_{m}$, are more deformed (smaller value of $s$ ) than with potentials $A_{g}$ and $B_{g}$, respectively.

The maximum overlap shown in Fig. 6 is always very close to 1 , always above 0.96 but typically around 0.98 or even higher. This indicates that the deformation described by Eqs. (29) and (30) works in general very well, although in some cases, for instance, potential $A_{m}$ with $b_{\text {ho }}=1.0$ (where the maximum overlap is about 0.96 ), a correction could probably be introduced.

The values of the computed scale parameters are shown in Fig. 7 as a function of $b_{\mathrm{ho}} / r_{2 \mathrm{D}}$. Together with them we show the estimate given in Eq. (37). More precisely, again exploiting the fact that in the case of the harmonic oscillator two-body potential we have that $b_{\mathrm{pp}}=r_{2 \mathrm{D}} \sqrt{3 / 2}$, we can then rewrite Eq. (37) as

$$
\frac{1}{s^{2}}=\sqrt{1+\frac{9}{4}\left(\frac{r_{2 \mathrm{D}}}{b_{\mathrm{ho}}}\right)^{4}},
$$

which is shown in Fig. 7 by the solid curve. As we can see, the estimate given above works very well for potentials $B_{g}$ and $B_{m}$ (squares in the figure), which correspond to the cases of well-bound 3D three-body states (Table II). In fact, for potential $B_{g}$, for which $\left|E_{3 \mathrm{D}}\right|$ is pretty large, the agreement is excellent. For the cases where the system is clearly less bound in three dimensions, potentials $A_{g}$ and $A_{m}$ (circles in the figure), the computed results disagree with the estimate in Eq. (39) in the region of intermediate squeezing. However, it is interesting to see how, for large squeezing, the computed curve for potentials $A_{g}$ and $A_{m}$ and the solid curve clearly converge. Therefore the estimate, (39), appears to be a very good way to determine the equivalence between $s$ and $b_{\text {ho }}$ also for potentials $\mathrm{A}_{g}$ and $\mathrm{A}_{m}$ in the cases of large squeezing, 

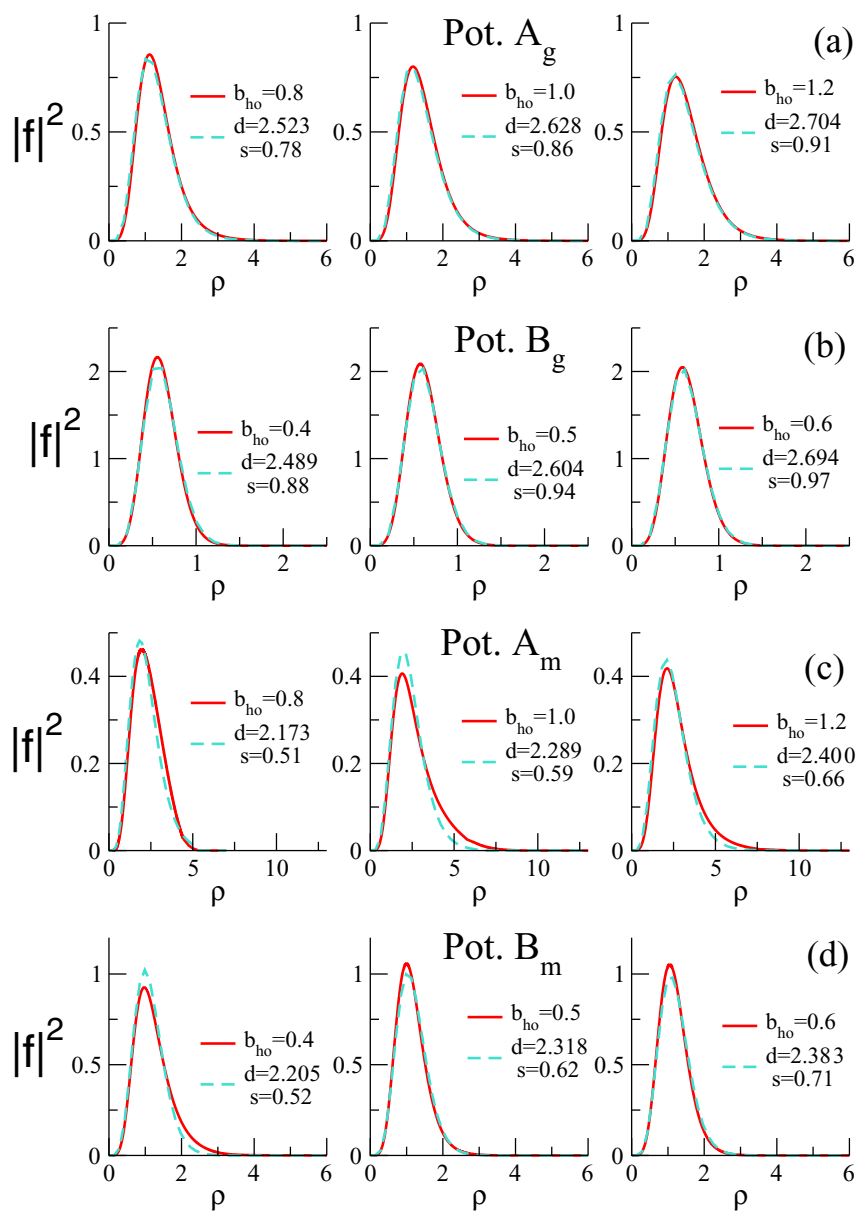

FIG. 8. The solid curves show, as a function of $\rho$, the square of the dominating radial wave functions, $f_{n}(\rho)$ in Eq. (16), for the potentials and external field parameters $b_{\text {ho }}$ shown in Fig. 6 (except the cases with $b_{\mathrm{ho}}=1.5$, which, for simplicity in the figure, have been omitted). Potentials (a) $A_{g}$, (b) $B_{g}$, (c) $A_{m}$, and (d) $B_{m}$. For each case, the dashed curve gives, also as a function of $\rho$, the square of the radial wave function $\tilde{f}_{n}^{(d)}(\rho, s)$ shown in Eq. (34), where the dimension $d$ provides the same ground-state energy as the calculation with the external field, and the scale parameter $s$, given in Fig. 6, maximizes the overlap $\mathcal{O}(s)$ in Eq. (32). The length unit is the range of the two-body interaction.

which, on the other hand, are the cases where the calculations with the external potential are more problematic.

Finally, let us directly compare the wave functions $\Psi_{\text {ext }}$ and $\tilde{\Psi}_{d}$. This can be done by simple comparison of the radial wave functions contained in the expansions Eqs. (16) and (33). Since the angular functions are the same in the two cases, the difference between the two wave functions will necessarily be contained in the radial functions $f_{n}(\rho)$ and $\tilde{f}_{n}^{(d)}(\rho, s)$.

In Fig. 8 we show the squares of the dominating radial wave functions, $f_{n}(\rho)$ (solid curves) and $\tilde{f}_{n}^{(d)}(\rho, s)$ (dashed curves), for some of the cases shown in Fig. 6 (in order to make the figure simpler cases with $b_{\text {ho }}=1.5$ have been omitted). In all of them, the dominating term provides more than $98 \%$ of the total norm. Figures $8(\mathrm{a})-8(\mathrm{~d})$ correspond to the results with potentials $A_{g}, B_{g}, A_{m}$, and $B_{m}$, respectively. The values of the dimension, $d$, and the scale parameter, $s$, are as indicated in Table III, i.e., the dimension, $d$, is such that the $d$ calculation gives the same ground-state energy as in the calculation with the external squeezing potential, and the scale parameter, $s$, is such that the overlap, $\mathcal{O}(s)$, in Eq. (32) is maximum.

As we can see in Fig. 8 the agreement is basically very good for all the cases shown. The largest discrepancy is observed for the Morse potential $\mathrm{A}_{m}$ with $b_{\text {ho }}=1.0$, which, as shown in Fig. 6, is the case with the smallest value of the maximum overlap, below 0.97 , between the two wave functions. In any case, the good agreement between the two radial wave functions illustrates how the wave function resulting from the $d$ calculation, after the appropriate reinterpretation in the ordinary $3 \mathrm{D}$ space, provides a very good description of the system squeezed by an external potential.

\section{SUMMARY AND CONCLUSIONS}

In this work the continuous squeezing of three-body systems from three to two dimensions has been investigated by means of two procedures: First, a method where the external confining potential is explicitly included; and, second, a method where the external potential does not enter, but instead, the dimension $d$ is allowed to take any intermediate value between $d=3$ and $d=2$.

The case of three identical spinless bosons with relative $s$ waves between all the particles is considered. For the twobody potentials we have used Gaussian and Morse radial forms whose scattering lengths vary from small, with a few fairly well-bound states, to rather large.

For the three-body calculations with an external field we have chosen the squeezing potential to be a one-body deformed harmonic oscillator acting on each of the three particles. The oscillator length in one of the dimensions is varied from $\infty$ (no squeezing) to a very small value corresponding to a fraction of the two-body interaction range. The deformation produced by the squeezing breaks the orbital angular momentum conservation, and the calculations must account for the resulting mixing of these quantum numbers. The consequence is that the adiabatic hyperspherical expansion must employ a large number of partial wave components in the limit where dimension 2 is approached.

The method with the noninteger dimension, $d$, as the parameter is formulated in terms of hyperspherical coordinates with spherical potentials and $s$ waves. The phase-space and centrifugal barrier potentials both correspond to the specified value of $d$. This method is technically precisely as simple as the ordinary three-body 3D computations without an external field.

With the two methods we have investigated how the ground-state energies vary when squeezing from three to two dimensions. Both methods show the same qualitative behavior, although the connection between the $2 \mathrm{D}$ and the $3 \mathrm{D}$ limits must be different in the two cases, since the oscillator lengths of the external potential and the dimension, $d$, vary in infinite and finite intervals, respectively. The three-body bound states do not necessarily have counterparts in the 3D and the $2 \mathrm{D}$ limits.

Comparison of the results from the two methods allows extraction of the function translating between $d$ and the 
oscillator length of the external squeezing potential. The knowledge of this function provides a subsequent prediction of which external field corresponds to a given $d$ value. To give us some insight, we have used a simplified system where harmonic oscillators (different to the external field) are used for the two-body interactions. In this way we have obtained an analytic expression relating the dimension $d$ and the oscillator length of the external field. This estimate is then compared to the results arising from the numerical computations. By choosing the rms radius of the three-body system in two dimensions as the length unit, we have found that the translational functions are remarkably similar to each other, as well as to the analytic function derived for the two-body oscillator potential.

To be able to predict any observable property entirely from $d$ calculations, we must also find a translation or interpretation providing the wave function corresponding to a $3 \mathrm{D}$ calculation with an external field. We naturally search for a deformed solution obtained from the spherical wave function in the $d$ method. This is achieved by scaling the squeezing coordinate relative to the two other coordinates. The scaling factor must depend on $d$, and it is defined such that the overlap between the two wave functions of equal energy from the two methods is maximum, preferentially unity.

This interpretation of the $d$-calculated wave function is suggested by the exact solution available when using oscillator interactions between the particles as well as for the external field. This analytic approximation is compared to the scaling parameter extracted numerically. The computed values are surprisingly close to the analytic curve for the potentials with well-bound three-body states in three dimensions, whereas they deviate when the three-body system is weakly bound in three dimensions. Comparing directly the wave functions from the two methods we find a remarkable agreement between them.

In summary, we have investigated in detail a new method to deal with three-body problems in noninteger dimensions between 2 and 3 . The basis is a $d$-dimensional phase space, a corresponding $d$-dependent generalized centrifugal barrier, and a spherical computation. The calculations are precisely as simple as the same three-body calculations in integer dimensions. We validate the method by comparing it to the brute force method in the ordinary 3D space. The noninteger dimension is simulated by a deformed external field, which effectively reduces movement in one coordinate from being free (in three dimensions) to zero (in two dimensions) space.

The equivalence of the two methods is shown for identical bosons by numerical calculations, where we provide a relatively accurate translation between the parameter $d$ and the external field length. This connection includes a prescription to obtain the complete deformed wave function from the $d$ calculation. Since the translation correctly reproduces the two limits of two and three dimensions, any prediction occurring for some $d$ value is bound to happen for some external field strength, which in turn is relatively precisely given by an analytic expression.

The presented $d$ method can be extended to asymmetric three-body systems with different mass ratios, to dimensions smaller than 2 and larger than 3, and perhaps to fermions and particle numbers larger than three.

\section{ACKNOWLEDGMENT}

This work has been partially supported by the Spanish Ministry of Science, Innovation and University MCIU/AEI/FEDER,UE (Spain) under Contract No. PGC2018-093636-B-I00.

\section{APPENDIX: $s$-WAVE SPHERICAL AND HYPERSPHERICAL HARMONICS IN $\boldsymbol{d}$ DIMENSIONS}

The angle-independent $s$-wave spherical harmonic in $d$ dimensions, $Y_{0}$, can be obtained by taking into account that the phase volume in $d$ dimensions is given by [14]

$$
\int d \Omega_{d}=\frac{2 \pi^{d / 2}}{\Gamma\left(\frac{d}{2}\right)}
$$

from which, since $\int Y_{0}^{*} Y_{0} d \Omega_{d}=1$, one immediately gets

$$
Y_{0}=\left[\frac{\Gamma\left(\frac{d}{2}\right)}{2 \pi^{d / 2}}\right]^{1 / 2} .
$$

Thus, the angular functions, $\Phi_{n}^{(d, i)}$, in Eq. (7) depend only on the hyperangle $\alpha=\arctan (x / y)$, where $\boldsymbol{x}$ and $\boldsymbol{y}$ are the usual Jacobi coordinates [11]. Typically, these angular functions are obtained as an expansion in terms of the hyperspherical harmonics, which for $d$ dimensions and relative $s$ waves, take the form [11]

$$
\mathcal{Y}_{K}^{(d)}(\alpha)=N_{\nu} P_{\nu}^{\left(\frac{d-2}{2}, \frac{d-2}{2}\right)}(\cos 2 \alpha) Y_{0} Y_{0},
$$

where $K=2 v$ is the hypermomentum, $N_{v}$ is the normalization constant, and $P_{v}^{\left(\frac{d-2}{2}, \frac{d-2}{2}\right)}$ is a Jacobi polynomial.

Finally, when solving the three-body problem it is always necessary at some point to rotate the hyperspherical harmonics, (A3), from one Jacobi set $i$ into a different set $j$. This is done by means of the Raynal-Revai coefficients, which for $s$ waves and $d$ dimensions are given by [11]

$$
\left\langle\mathcal{Y}_{K}(i) \mid \mathcal{Y}_{K^{\prime}}(j)\right\rangle=\delta_{K K^{\prime}} \frac{P_{\nu}^{\left(\frac{d-2}{2}, \frac{d-2}{2}\right)}\left(\cos 2 \gamma_{i j}\right)}{P_{\nu}^{\left(\frac{d-2}{2}, \frac{d-2}{2}\right)}(1)},
$$

where the angle $\gamma_{i j}$ is given, for instance, in Ref. [11].
[1] I. Bloch, J. Dalibard, and W. Zwerger, Rev. Mod. Phys. 80, 885 (2008).

[2] S. Deng, Z.-Y. Shi, P. Diao, Q. Yu, H. Zhai, R. Qi, and H. Wu, Science 353, 371 (2016).
[3] J. R. Armstrong, A. G. Volosniev, D. V. Fedorov, A. S. Jensen, and N. T. Zinner, J. Phys. A: Math. Theor. 48, 085301 (2015).

[4] F. S. Møller, D. V. Fedorov, A. S. Jensen, and N. T. Zinner, J. Phys. B: At. Mol. Opt. Phys. 52, 145102 (2019). 
[5] M. T. Yamashita, F. F. Bellotti, T. Frederico, D. V. Fedorov, A. S. Jensen, and N. T. Zinner, J. Phys. B: At. Mol. Opt. Phys. 48, 025302 (2015).

[6] J. H. Sandoval, F. F. Bellotti, A. S. Jensen, and M. T. Yamashita, J. Phys. B: At. Mol. Opt. Phys. 51, 065004 (2018).

[7] D. S. Rosa, T. Frederico, G. Krein, and M. T. Yamashita, Phys. Rev. A 97, 050701(R) (2018).

[8] J. Levinsen, P. Massignan, and M. M. Parish, Phys. Rev. X 4, 031020 (2014).
[9] E. Garrido, A. S. Jensen, and R. Álvarez-Rodríguez, Phys. Lett. A 383, 2021 (2019).

[10] E. Garrido and A. S. Jensen, Phys. Rev. Res. 1, 023009 (2019).

[11] E. Nielsen, D. V. Fedorov, A. S. Jensen, and E. Garrido, Phys. Rep. 347, 373 (2001).

[12] A. S. Jensen, K. Riisager, D. V. Fedorov, and E. Garrido, Rev. Mod. Phys. 76, 215 (2004).

[13] E. Garrido, Few-Body Syst. 59, 17 (2018).

[14] S. I. Hayek, Advanced Mathematical Methods in Science and Engineering (Marcel Dekker, New York, 2001), p. 645. 